\title{
Comparison of active and passive EMI filters to reduction conducted noise
}

\author{
Marian Pasko ${ }^{1}$, Marek Szymczak ${ }^{1, *}$ \\ ${ }^{1}$ Silesian University of Technology, Faculty of Electrical Engineering, 44-100 Gliwice, Poland
}

\begin{abstract}
The article presents the results of experimental studies of passive and active EMI filters for reduction of conducted noise. Firstly, the article presents selected structures of passive filters that have been tested in terms of insertion loss parameter describing their efficiency. Then, active filters were used to improve the efficiency of insertion loss of passive filters under the same conditions. Finally, the results and conclusions are drawn from the measurements of both types of filters for the reduction of conducted noise.
\end{abstract}

\section{Introduction}

Electric power systems used in electric cars use the power supply voltage (from $200 \mathrm{~V}$ to $550 \mathrm{~V}$ ) keyed at high frequency (up to $200 \mathrm{kHz}$ ) which cause high level of EMI noise [1]. The limitation of the level of EMI is restricted by IEC/CISPR standards (EN 55025: 2008) which force the construction of larger and more complex filters what directly increases their cost [2]. Therefore, research is being conducted on the possibility of using active solutions to suppress EMI noise by replacing passive filters or significantly improving their properties [3].

\section{EMI filters}

Two passive filters and a combination of a passive and an active EMI filter were evaluated in this article. Attention was focused on measurements of common mode EMI noise, whose currents propagate in both supply lines + and - in the same direction and close through the PE grounding wire.

\subsection{Passive EMI filters}

$\mathrm{CM}$ inductors, in order to have high efficiency in suppressing CM EMI, must have high inductance. This can be achieved in two ways, by increasing the number of turns or by using high permeability ferromagnetic materials. In the case of using bus bars for power distribution in automotive, the problem is the winding of several turns on the core using copper flat bars, thus leading to using cores made of expensive ferromagnetic materials (e.g. nanocrystalline [4]) or increasing the number of cores.

In this chapter, an insertion losses study of two passive filters was carried out using cores of nanocrystalline material with different magnetic permeability. Figure 1 presents a schematic of the measurement stand proposed by the authors.

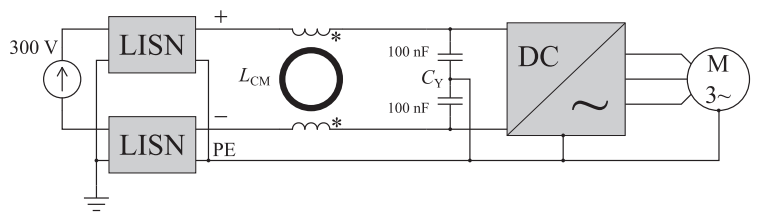

Fig. 1. Schematic diagram of the proposed measuring station for passive filters.

The first core tested was Hitachi FT-3K50T with one turn. The resulting $L_{\mathrm{CM}}$ choke had an inductance of about $2 \times 50 \mu \mathrm{H}$. The attenuation properties of the resulting filter (1 turn) depend only on the magnetic and geometric properties of the core. The second tested core was W424-04 from VAC. The choke is made of 4 nanocrystalline cores with a total inductance value of 2x $600 \mu \mathrm{H}$.

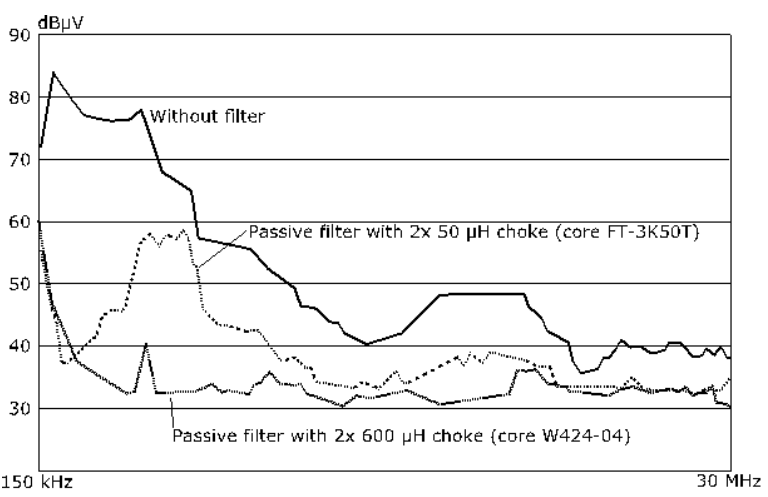

Fig. 2. Obtained spectra of CM EMI generated by inverter without filter, with passive filter with core FT-3K50T and W424-04.

Figure 2 presents a comparison of obtained spectra of CM EMI generated by the power inverter with a passive filter with core FT-3K50T and W424-04. When

\footnotetext{
Corresponding author: Marek.Szymczak@polsl.pl
} 
comparing both filters in terms of attenuation, one can notice a significant increase in attenuation for the W424-04 core, which is due to the fact that the choke built on it has a much higher inductance value.

\subsection{Active EMI filters}

The article focuses on the group of feedback filters whose operation consists in creating a compensating signal to suppress the ripples generated by the source (closed loop).

Figure 3 presents a diagram of a measurement stand proposed by the authors, in which a type III active filter (voltage detection and current cancelation) was applied.

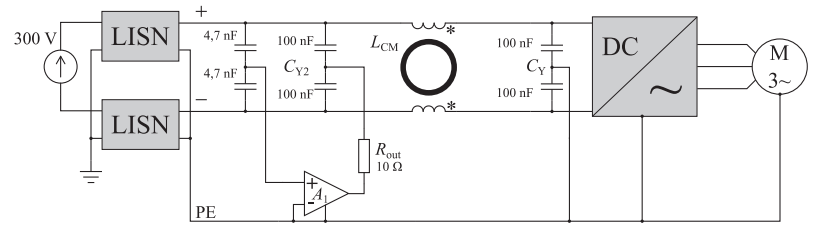

Fig. 3. Schematic diagram of the proposed measuring stand for active EMI filters.

This filter is a cascade connection of a passive filter (with a coil on the FT-3K50T core with a inductance of $2 \times 50 \mu \mathrm{H}$ ), and a current cancelation active filter. The core used is the same as in the previously constructed passive filter, and the amplifier has a gain of $A_{1}=100 \mathrm{~V} / \mathrm{V}$. The spectrum of CM EMI that has been registered with the analyzer is shown in Figure 4.

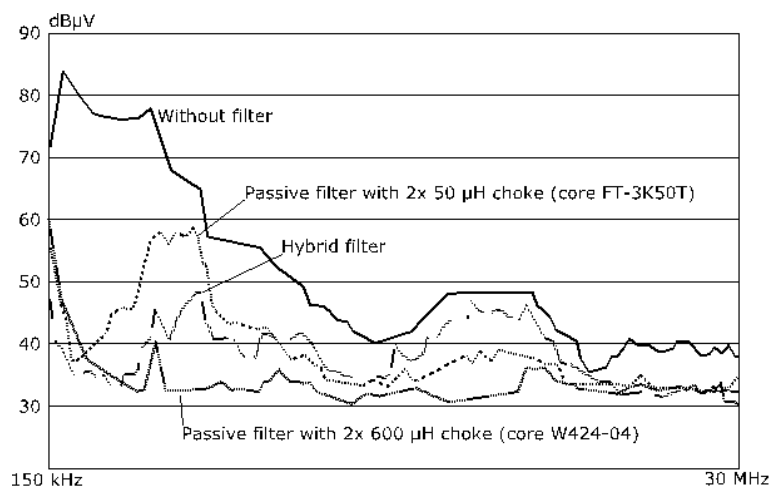

Fig. 4. Obtained spectra of CM EMI generated by inverter without filter, with passive filter with core FT-3K50T and W424-04, and cascade combination of active and passive filters.

When comparing the obtained spectrum with the spectrum after suppression with the passive filter itself with the FT-3K50T core $(2 \times 50 \mu \mathrm{H})$, one can notice that the attenuation of the hybrid filter is $20 \mathrm{~dB}$ higher in the range of $150 \mathrm{kHz}-6 \mathrm{MHz}$.

\section{Summary}

The article investigates two passive filters and one cascade connection of a passive and an active filter. Passive filters were built using two different cores (nanocrystalline) with different permeabilities, obtaining extremely different inductance values of $L_{\mathrm{CM}}$. This experiment was used to check what effect the inductance of inductors has on filter attenuation.

In the case if a filter with the inductor of $2 \times 50 \mu \mathrm{H}$, based on the FT-3K50T core, attenuation was obtained from $10 \mathrm{~dB}$ to $40 \mathrm{~dB}$ depending on the frequency. The filter with an inductor of $2 \times 600 \mu \mathrm{H}$ inductance, in which the W424-04 core was used, had an insertion loss modulus of $30 \mathrm{~dB}$ to $50 \mathrm{~dB}$. The difference in attenuation results mainly from different inductance values, and for a filter in which the inductor has a higher inductance, the insertion loss modulus is higher.

The next tested system was a cascade of a passive and an active filter, in which the $L_{\mathrm{CM}}$ inductor had the inductivity of $2 \times 50 \mu \mathrm{H}$, and a type III active filter (voltage detection and current cancelation). The obtained insertion loss modulus, depending on the frequency, reached even $60 \mathrm{~dB}$, in the frequency range from $150 \mathrm{kHz}$ to $6 \mathrm{MHz}$. For higher frequencies (from $15 \mathrm{MHz}$ to $24 \mathrm{MHz}$ ) there was a slight amplification effect in relation to the passive filter itself. The insertion loss modulus of the passive filter itself in this band was about $10 \mathrm{~dB}$, whereas the cascade connection showed a loss rate of only from $1 \mathrm{~dB}$ to $5 \mathrm{~dB}$. This is caused by the phase shift in the active filter due to the limitation of the frequency response of the $A_{1}$ amplifier.

When comparing a passive filter (with an $L_{\mathrm{CM}}$ inductor of $2 \times 600 \mu \mathrm{H}$ inductance) with a cascade combination of a passive and an active filters, it can be noticed that in the frequency band from $150 \mathrm{kHz}$ to $4 \mathrm{MHz}$ similar attenuation values were obtained. This allows us to state that by using a choke of lower inductivity, made of a material with lower magnetic permeability, but adding an active filter system, an insertion loss modulus can be obtained at a similar level to a filter with a higher inductance choke. Thanks to this, one can use a cheaper ferromagnetic material with worse parameters, thus reducing the cost of the filter, its weight and dimensions.

\section{References}

1. M. Böh, A. Lohner, N. El Amrani, Efficiency increasing by a variable DC link voltage in combination with a bang-bang controlled inverter for an automotive application PCIM Europe (2017)

2. S. Schefler, C. Keller, K. Spanos, S. Weber, Systemsimulations with EMI-Filter in an Automotive HighVolt Environment PCIM Europe (2017)

3. M. Heldwein, H. Ertl, J. Biela, J. Kolar, IEEE Trans. On Industrial Electron. 57, 5 (2010)

4. P. Cantillon-Murphy, T. C. Neugebauer, C. Brasca, D. J. Perreault, IEEE Power Electron. Letters 2, 2 (2004) 